\title{
New method for rapid Susceptibility Testing on blood culture with HB\&L system: preliminary data
}

\author{
Vincenzo Rondinelli, Stefania Giglio, Rosanna Masciari \\ Microbiologia e Virologia Azienda Ospedaliera Pugliese-Ciaccio, Catanzaro
}

Key words: Sepsis diagnosis, Blood culture, Turn around time, Therapeutic turn around time, Antimicrobial susceptibility test

Nuova metodica per l'esecuzione rapida dell'antibiogramma nelle emocolture mediante sistema HB\&L: dati preliminari

\section{SUMMARY}

Blood culture, although represents the gold standard in detecting the ethiological agent of sepsis, is rather rarely required in relation to the real diagnostic importance.

The result of this test depends in fact on many factors (sample volume, time of collection, accuracy, antibiotic therapy, contamination, number of drawings, drawing site, interpretation difficulties, etc.) that are often considered by many clinicians so limited as to doubt about their actual value.

The disadvantages are therefore represented by the lack of standardization but also by the low sensitivity and above all by the technical times too long for the clinical needs.

Blood culture begins with the drawing of samples from the "septic" patient followed incubation of the bottles in automatic thermostated systems. In case of positive result ( 36 hours), the culture is Gram stained and streaked on solid media in order to obtain isolated colonies for the identification and the susceptibility testing (48 hours from positive result).

The long time required for pathogen identification and susceptibility testing involves empirical broad spectrum antibiotic therapy that can promote the increase of bacterial resistance but also patient management costs.

A clinically useful report should be available on short notice in order to guide the clinician to choose the most appropriate antibiotic.

The microbiologist has therefore the hard work of reviewing the organization and the management of the procedures. We have therefore started to consider the possibility of treating the blood as an biological liquid in order to quickly determine the susceptibility of bacteria to antibiotics.

\section{INTRODUZIONE}

L'emocoltura, malgrado rappresenti tuttora il gold standard nella rilevazione dell'agente eziologico della sepsi, è un esame di laboratorio che viene richiesto in quantità ridotta in rapporto alla reale importanza diagnostica.

Il suo risultato dipende, infatti, da tantissimi fattori (il volume del campione, il momento del prelievo, la sua accuratezza, la terapia antibiotica in corso, la contaminazione del campione, il numero di prelievi per evento settico, la sede di prelievo, le difficoltà interpretative, ecc.) che spesso sono considerati da molti clinici così limitanti da metterne in discussione l'effettivo valore.

Gli svantaggi sono rappresentati dunque dalla mancata standardizzazione ma anche dalla bassa sensibilità e soprattutto dai tempi tecnici troppo lunghi rispetto alle necessità cliniche. Il diagramma di flusso dell'emocoltura inizia con l'esecuzione dei prelievi al paziente con sospetta "sepsi" ed il loro invio in laboratorio e prosegue con l'incubazione dei flaconi nei sistemi automatici termostatati.

In caso di positività (mediamente 36 ore) si procede con la colorazione di Gram per valutare l'affinità tintoriale e la morfologia dei batteri, con la semina sui terreni per ottenere le colture in piastra ed infine con l'identificazione e l'antibiogramma (mediamente altre 48 ore dalla segnalazione di positività). I lunghi tempi necessari per l'identificazione del patogeno e l'esecuzione del test di sensibilità implicano una terapia antibiotica empirica, ad ampio spettro, che può favorire non solo l'aumento delle resistenze batteriche ma anche quello dei costi di gestione dei pazienti.

Un referto clinicamente utile deve invece essere disponibile in tempi ristretti per poter fornire al clinico tutte le informazioni utili per instaurare una terapia antibiotica adeguata nel più breve tempo possibile.

Il microbiologo ha dunque il gravoso compito di rivedere organizzazione e gestione delle procedure (5) e abbiamo pertanto iniziato a muoverci in tale direzione valutando la possibilità di trattare il sangue alla stregua di un liquido biologico al fine di determinare rapidamente la sensibilità dei batteri agli antibiotici.

\section{MATERIALI E METODI}

L'esame colturale rapido delle urine e degli altri liquidi biologici è eseguito, nel nostro laboratorio, con lo strumento HB\&L della ditta ALIFAX (Padova, Italia).

Lo strumento automatico permette di rilevare l'intensa attività di replicazione dei batteri grazie alla tecnologia "Light Scattering" che garantisce, in un brodo di arricchimento eugonico, una sensibilità di lettura 100 volte superiore ai sistemi fotometrici. Consente inoltre di rappresentare graficamente la cinetica di crescita batterica, esprimendone la carica in CFU/ml (Colony Forming Unit).

$500 \mu \mathrm{l}$ di liquido biologico e $200 \mu \mathrm{l}$ di DEB, un supplemento di coltura ricco di sostanze nutritive, vengono inoculati in un apposito flacone contenente un terreno liquido specifico; il flacone viene posto nello strumento ed i risultati quantitativi della carica batterica presente nei liquidi biologici sono disponibili mediamente in 2-3 ore.

Per favorire una maggiore comprensione del quadro clinico il sistema può effettuare, contemporaneamente al test colturale, quello per il Potere Antimicrobico Residuo (PAR test) (3). Lo strumento può testare immediatamente il campione positivo con un pannello personalizzato di antibiotici secondo le linee guida in uso presso i vari reparti ospedalieri.

Gli antibiotici attualmente disponibili sono: Amoxicillinaacido clavulanico, Ampicillina G. Vancomicina, Piperacillina, Piperacillina Tazobactam, Ceftazidime, Cefuroxime, Ceftriaxone, Aztreonam, Fosfomicina, Linezolid, Cefotaxime, Norfloxacina, Levofloxacina, Ciprofloxacina, Cotrimoxazolo, Amikacina, Gentamicina, Oxacillina (SNC) ed Oxacillina (aureus).

L'antibiogramma clinico, disponibile in 3 ore, è stato messo a punto seguendo le linee guida del CUMITECH (1) e CLSI. Diversi studi comparativi verso il sistema di riferimento Kirby Bauer dimostrano un'alta correlazione dei risultati (6). 
I risultati sono espressi in PIC (Percentuale di Inibizione della Crescita).

\section{Emocolture}

I flaconi contenenti il sangue vengono incubati nello strumento automatico Bactec ${ }^{\circledR} 9120$ della BD, che agisce sia da termostato che da rilevatore della $\mathrm{CO}_{2}$ prodotta dalla crescita di microrganismi.

Le emocolture positive vengono seminate in terreni generali, selettivi e differenziali per l'isolamento del patogeno che viene poi identificato e sottoposto al test di sensibilità in vitro con i pannelli NMIC/ID-88 per i Gram negativi, PMIC/ID-73 per Gram positivi ed enterococchi e SMIC/ID-9 per gli streptococchi, forniti dalla ditta BD ed utilizzati sullo strumento Phoenix ${ }^{\mathrm{TM}}$.

I ceppi batterici utilizzati per il controllo di qualità sono Escherichia coli $\mathrm{ATCC}^{\circledR} 25922^{\mathrm{TM}}$, Pseudomonas aeruginosa ATCC $^{\circledR} 27853^{\mathrm{TM}}$ e Staphylococcus aureus ATCC $^{\circledR} 29213^{\mathrm{TM}}$, forniti dalla ditta Oxoid.

\section{Antibiogramma ALIFAX}

Abbiamo condotto su un campione di oltre 100 emocolture (circa $2 / 3$ positive per batteri Gram+ ed $1 / 3$ per batteri Gram-) numerosissime prove per trovare la diluizione ottimale del contenuto del flacone di emocoltura risultata positiva, che consentisse, nei tempi più rapidi possibili, il raggiungimento della crescita idonea a determinare il Mc Farland richiesto (0.5) per l'antibiogramma clinico.

Questo viene eseguito seminando $100 \mu \mathrm{l}$ della sospensione batterica allo $0.5 \mathrm{Mc}$ Farland in ogni flacone contenente 100 $\mu \mathrm{l}$ dei vari antibiotici ricostituiti dallo stato liofilo. La PIC viene evidenziata dallo strumento o con crescita della curva batterica (Resistente: R) o con assenza di curva di crescita (Sensibile: S) o con accenno di curva (Intermedio: I), sempre comunque con valori percentuali. Con lo strumento HB\&L viene anche saggiata la curva di riferimento della PIC, ricavata utilizzando $100 \mu \mathrm{l}$ della sospensione batterica in un flacone di brodo eugonico privo di antibiotici (7).

Le molecole antibiotiche da noi utilizzate nello studio sono state scelte, in base alle esigenze dei clinici dei reparti interessati quali rianimazione, ematologia, patologia neonatale, medicina d'urgenza, cardiochirurgia, in cui la tempistica della terapia mirata risulta determinante.

Nel nostro studio i due tipi di antibiogramma sono stati utilizzati in parallelo per verificare l'attendibilità dei risultati, la loro riproducibilità e l'affidabilità della PIC nei confronti della MIC.

\section{RISULTATI}

La ricerca della diluizione ottimale per ottenere nei tempi più rapidi possibili la crescita batterica per lo $0.5 \mathrm{Mc}$ Farland è stata molto lunga ed impegnativa.

Il primo tentativo è stato effettuato utilizzando $500 \mu \mathrm{l}$ di contenuto del flacone di emocoltura positiva e $200 \mu \mathrm{l}$ di DEB, ma lo strumento sistematicamente ha segnalato la torbidità del campione non riuscendo a evidenziare la replicazione batterica (Figura I).

Anche con $500 \mu$ della diluizione 1:2 del contenuto del flacone con soluzione fisiologica e $200 \mu \mathrm{l}$ di DEB si sono ottenuti risultati analoghi.

Con $500 \mu \mathrm{l}$ della diluizione 1:4 del contenuto del flacone con soluzione fisiologica e $200 \mu \mathrm{l}$ di DEB lo strumento non ha mai segnalato la torbidità del campione, ha iniziato a segnalare la crescita batterica, ma quasi sempre il campione è risultato anomalo (Figura II).

L'uso di $500 \mu$ della diluizione 1:8 del contenuto del flacone con soluzione fisiologica e $200 \mu \mathrm{l}$ di DEB non ha messo in evidenza sostanziali modifiche; il campione non è segnalato ano-

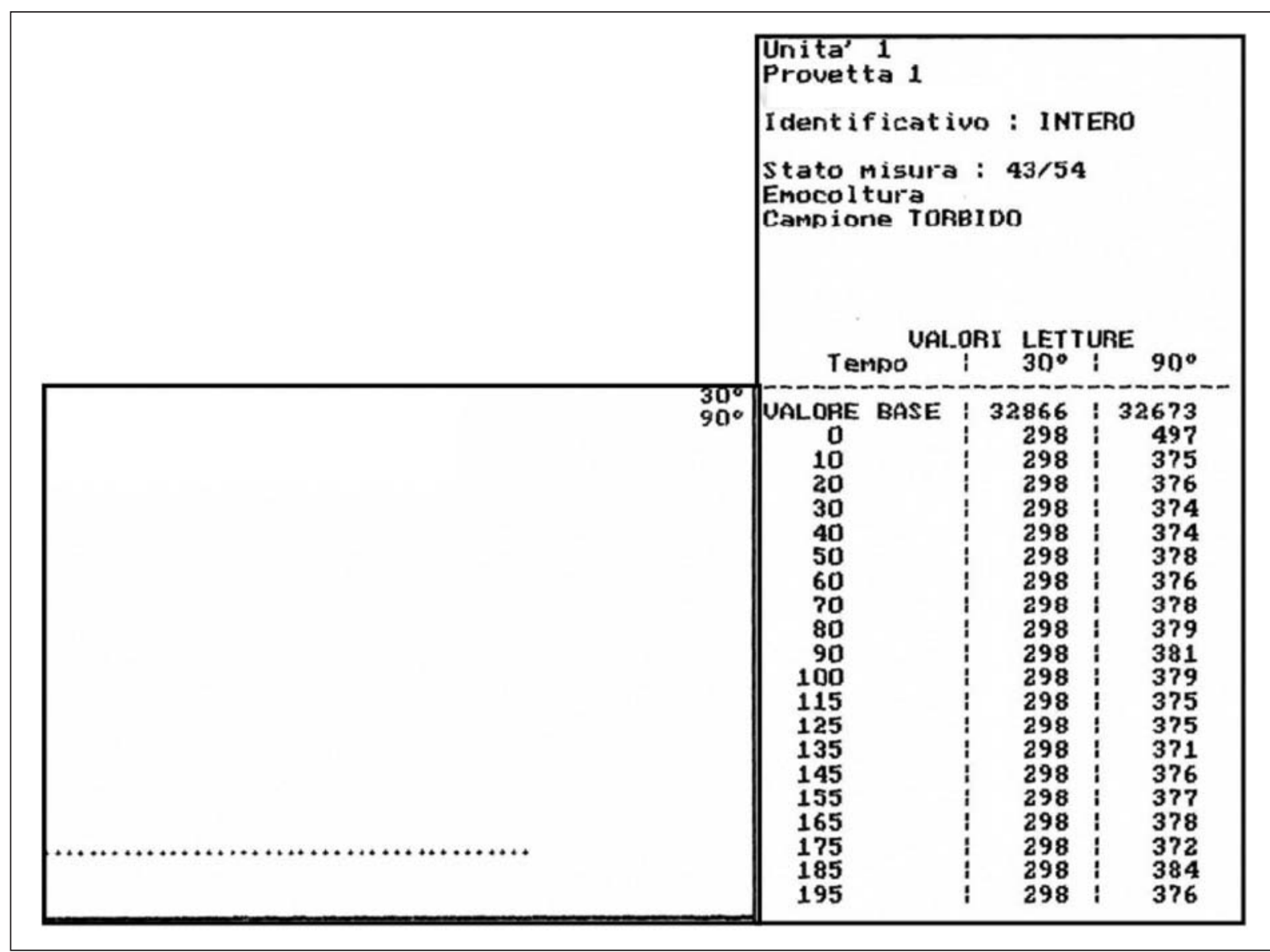

Figura I. Lettura con HB\&L del campione indiluito. 
malo, lo strumento ha segnalato meglio la crescita batterica, ma non sistematicamente e con una fase di latenza iniziale. $500 \mu \mathrm{l}$ della diluizione 1:10 del contenuto del flacone con soluzione fisiologica e $200 \mu$ di DEB rappresenta sistematicamente la diluizione ottimale che ci consente in circa 30 minuti di avere la crescita idonea (Figura III) per il raggiungimento del Mc Farland richiesto per l'antibiogramma clinico.

Non abbiamo riscontrato, a tale diluizione, differenze rilevanti tra germi Gram+ e Gram- in termini di crescita batterica.

Abbiamo comunque valutato anche la diluizione 1:12 ma non abbiamo riscontrato variazioni significative; diluizioni ancora maggiori $(1: 14,1: 16,1: 20)$ si sono rivelate inadatte perché l'eccessiva diluizione non consente di acquisire risultati utili.Anche la comparazione dell'antibiogramma clinico con quello classico fornito dallo strumento Phoenix ci ha notevolmente impegnato ed i risultati ottenuti sono nel complesso da ritenere sovrapponibili, essendo i casi discordanti statisticamente poco significativi.

Gli antibiotici testati, su richiesta dei clinici, sono prevalentemente Amikacina, Piperacillina- Tazobactam e Levofloxacina per i Gram negativi e Vancomicina, Linezolid e Oxacillina per i Gram positivi. Riportiamo, come esempio, il confronto tra l'antibiogramma fornito dal Phoenix ${ }^{\mathrm{TM}}$ per un paziente ematologico con un emocoltura positiva per Klebsiella pneumoniae (Figura IV) e l'antibiogramma clinico condotto saggiando rispettivamente l'Amikacina (Figura V) e la PiperacillinaTazobactam (Figura VI).

L'antibiogramma clinico fornisce dunque indicazioni terapeutiche valide: la Piperacillina-Tazobactam non è certamente, valutando la MIC riportata nel referto del Phoenix, l'antibiotico di prima scelta, e tale si conferma anche con la PIC fornita dall'HB\&L, che riscontra una percentuale di resistenza del 7\%.

\section{DISCUSSIONE E CONCLUSIONI}

Il tempo analitico, nella diagnosi della sepsi, è di importanza fondamentale al fine di intervenire con successo sia sul Turn Around Time (TAT: tempo che intercorre tra il momento del prelievo e l'acquisizione del risultato) delle emocolture sia sul Therapeutic Turn Around Time (TTAT: intervallo di tempo tra il momento della richiesta e quello in cui il risultato consente al medico di reparto di prendere una decisione di valore clinico) $(2,4)$.

Abbiamo pertanto constatato che l'utilizzo della metodica sopra descritta consente di soddisfare pienamente queste necessità mantenendo un livello di affidabilità in linea con le metodiche più diffuse in commercio.

I tempi per la messa a punto sono stati lunghi ma, ad oggi, la metodica, sotto il profilo procedurale, risulta essere semplice e con ridotte possibilità di errore.

Lo strumento HB\&L permette di effettuare un antibiogramma mirato in funzione delle diverse necessità cliniche del singolo paziente, senza necessità di utilizzare pannelli preformati, riducendo i costi e fornendo una risposta specifica.

Abbiamo riscontrato una buona correlazione con il metodo in uso (Phoenix $\left.{ }^{\mathrm{TM}} \mathrm{BD}\right)$.

La possibilità di verificare la sensibilità del germe a ciascun antibiotico testato, anche graficamente (Figure V e VI), fornisce al clinico ulteriori informazioni riguardo alla cinetica di crescita dei batteri.

Con l'introduzione di nuovi antibiotici, attualmente in fase di validazione, quali ad esempio Teicoplanina, Meropenem e Clindamicina migliorerà la prospettiva di raggiungimento del successo terapeutico.

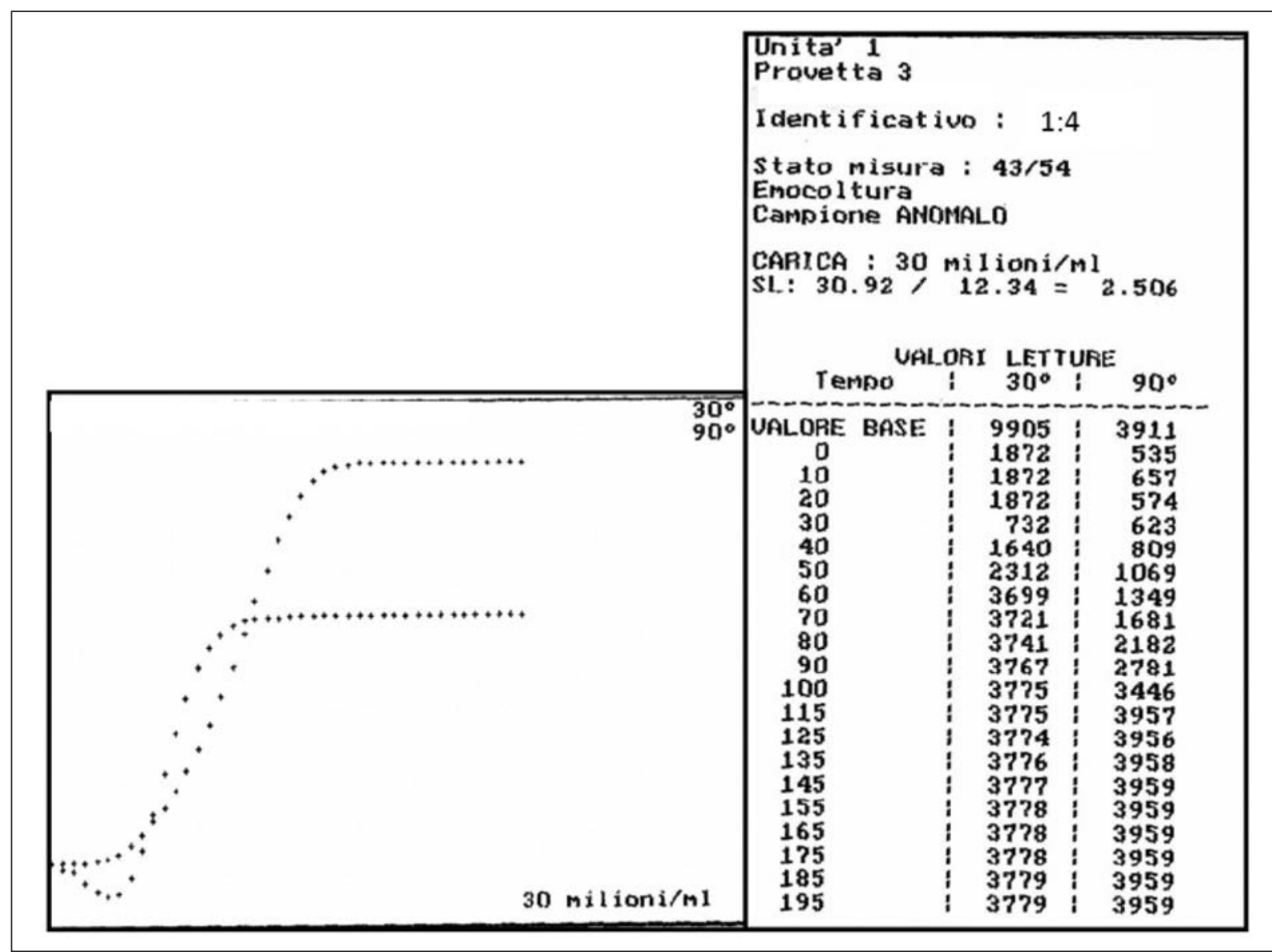

Figura II. Lettura con HB\&L del campione diluito I:4. 


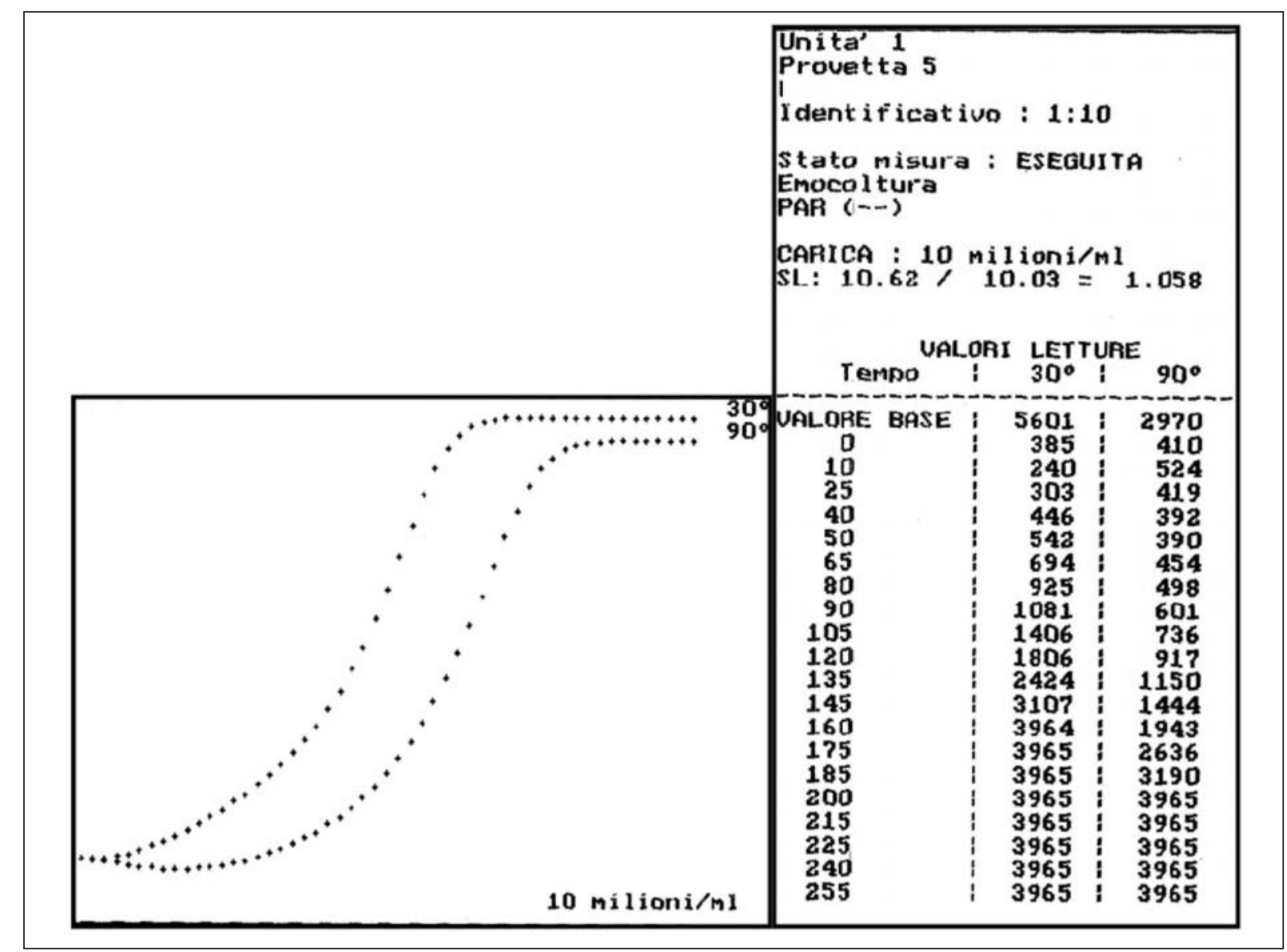

Figura III. Lettura con HB\&L del campione diluito I:I0.

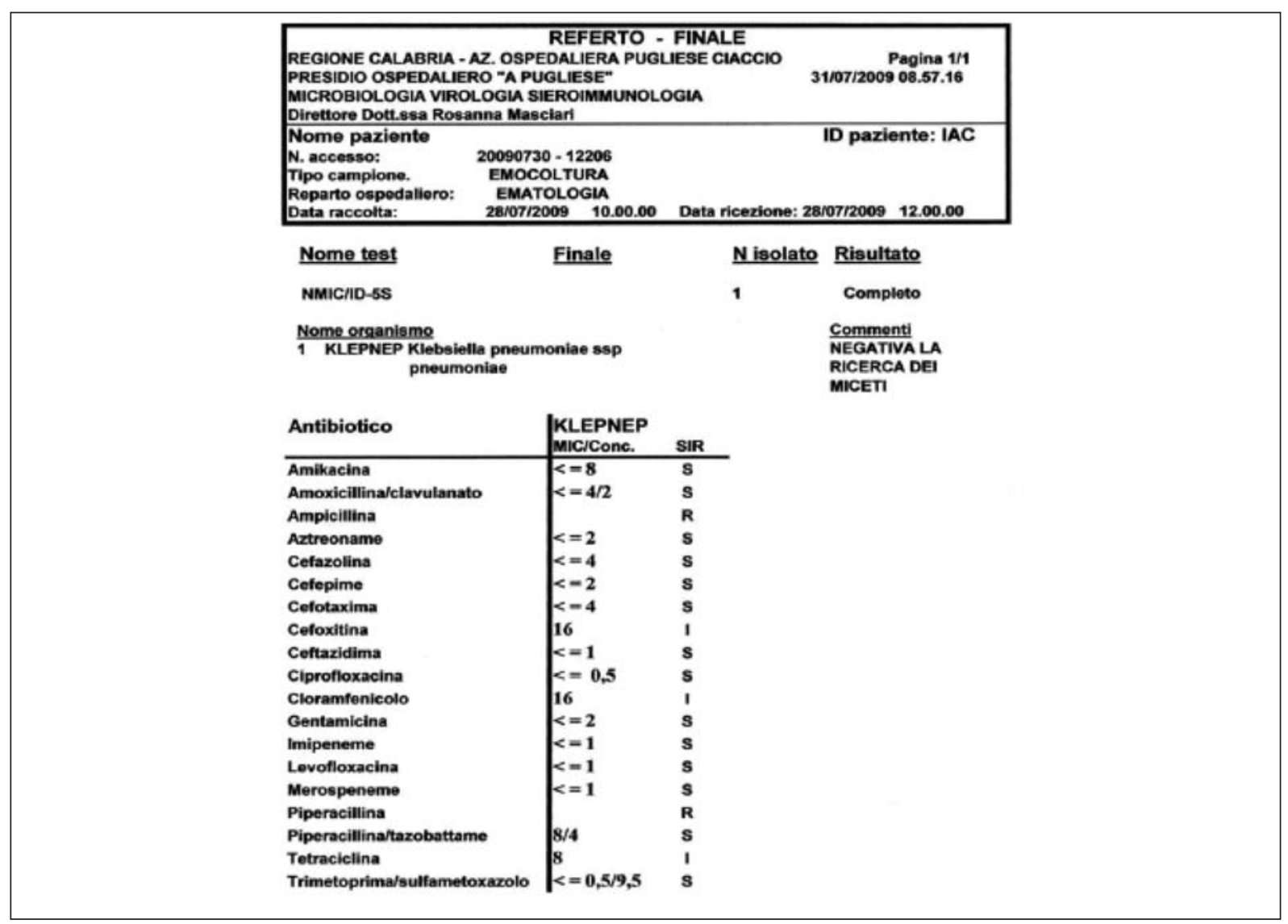

Figura IV. Antibiogramma (MIC) con Phoenix. 


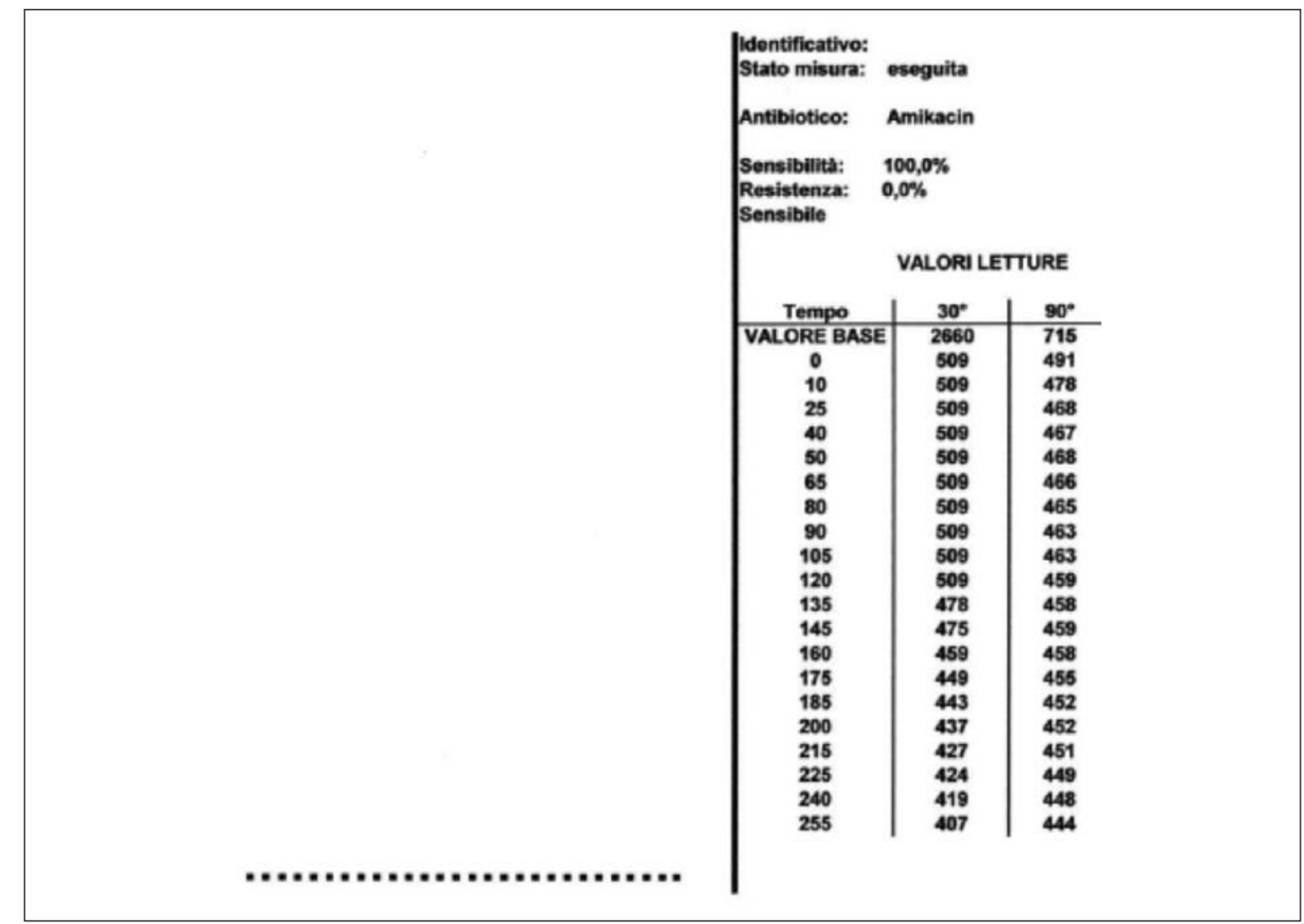

Figura V. Antibiogramma Amikacina (PIC) con HB\&L.

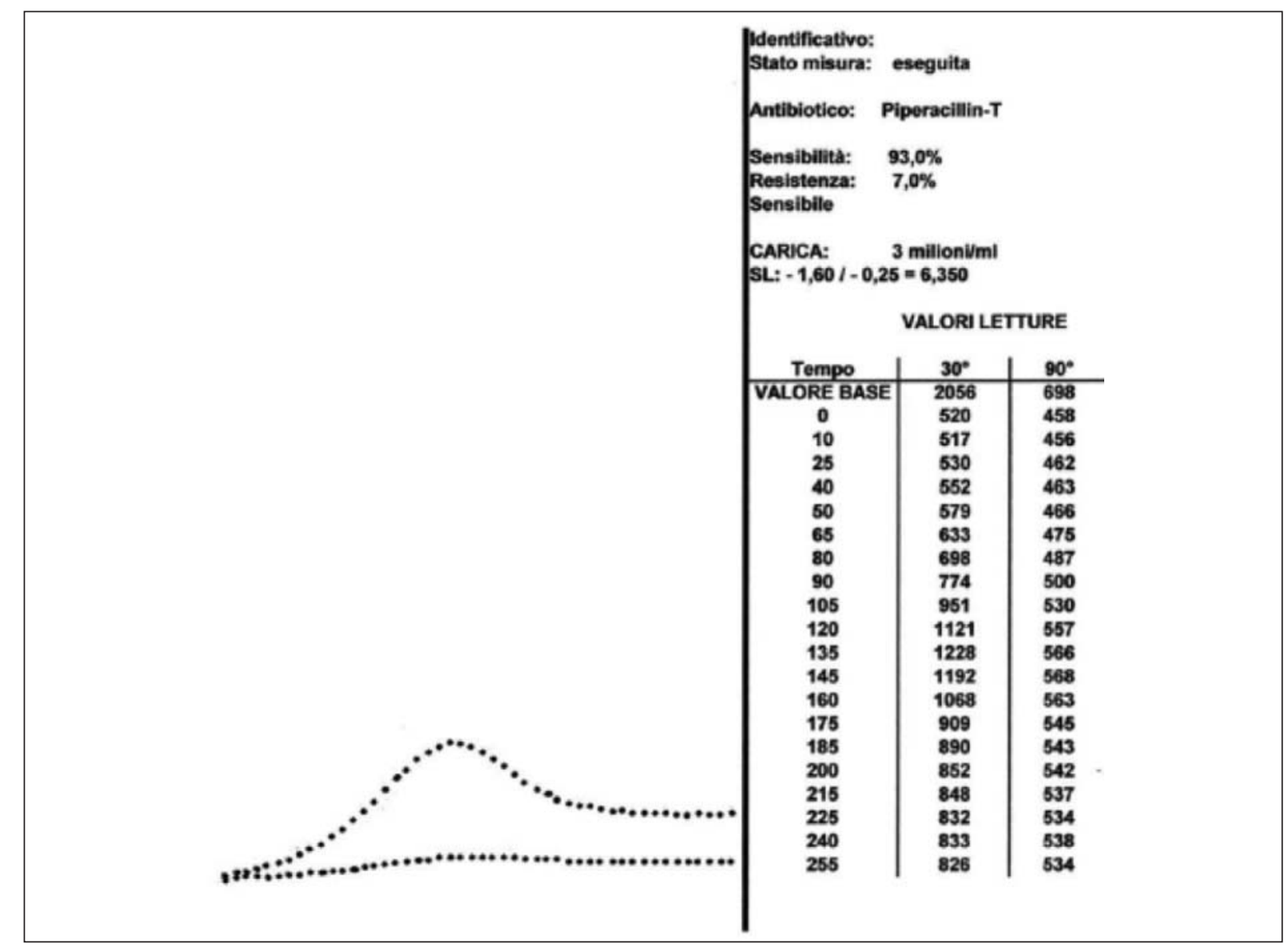

Figura VI. Antibiogramma Piperacillina Tazobactan (PIC) con HB\&L. 


\section{BIBLIOGRAFIA}

1. Clarridge JE, Johnson JR, Pezzo MT. Laboratory diagnosis of urinary tract infections. Cumitech November 1998.

2. Fiel S. Guidelines and critical pathways for severe hospital acquired pneumonia. FCCP Chest 2001, 119: 412-8

3. Fontana C, Favaro M, Minelli S, Bossa MC, Altieri A, Favalli C. A novel culturing system for fluid samples. Med Sci Monit 2009; 15 (2): 55-60.

4. Funke G, Funke-Kissling P. Use of the BD Phoenix automated microbiology system for direct identification and susceptibility testing of Gram negative rods from positive blood cultures in a three-phase trial. J Clin Mic,
2004; 42 (4): 1466-70

5. Kroumova V, Gobbato E, Macaluso P, et al. Preliminary indications for antibiotic susceptibility tests in less than six hour in positive blood cultures. Microb Med 2010; 25 (1): 24-6.

6. Roveta S, Debbia EA, Marchese A. Antibiotic susceptibility tests of uropathogens using the Uro-Quick System directly on urine in comparison with the Kirby-Bauer method. GIMMOC 2003; VII (2): 67-74.

7. Roveta S, Debbia EA, Marchese A. Evaluation of the Uro-Quick, a new rapid automated system, for the detection of well-characterized antibiotic resistant bacteria. Chemother 2004; 16 (1): 76-87. 